PROCEEDINGS OF THE

AMERICAN MATHEMATICAL SOCIETY

Volume 129, Number 11, Pages 3177-3184

S 0002-9939(01)06038-5

Article electronically published on April 2, 2001

\title{
A GENERALIZATION OF THE LIPSCHITZ SUMMATION FORMULA AND SOME APPLICATIONS
}

\author{
PAUL C. PASLES AND WLADIMIR DE AZEVEDO PRIBITKIN \\ (Communicated by Dennis A. Hejhal)
}

\begin{abstract}
The Lipschitz formula is extended to a two-variable form. While the theorem itself is of independent interest, we justify its existence further by indicating several applications in the theory of modular forms.
\end{abstract}

\section{LIPSCHITZ'S FORMULA}

As originally conceived, the Lipschitz Summation Formula (henceforth, $L S F$ ) gives a Fourier expansion for certain functions which arise in the theory of modular forms. More explicitly, it states that if $\mu \in \mathbb{Z}$ and $\operatorname{Re} \alpha>1$ or $\mu \in \mathbb{R} \backslash \mathbb{Z}$ and $\operatorname{Re} \alpha>0$, then

$$
\sum_{m=-\infty}^{\infty} \frac{e^{-2 \pi i \mu m}}{(\tau+m)^{\alpha}}=\frac{(-2 \pi i)^{\alpha}}{\Gamma(\alpha)} \sum_{n+\mu>0}(n+\mu)^{\alpha-1} e^{2 \pi i(n+\mu) \tau},
$$

for $\operatorname{Im} \tau>0$ [9]. There are several methods of proof, the most common being by Poisson summation (see 1 for a statement of the Poisson summation formula and [5, pp. 65-72] for a complete proof of the Lipschitz formula in the case $\mu=0$, $\alpha>1$ using contour integration). The LSF implies the functional equation for the Riemann zeta-function [6]. In number theory, the LSF is a useful tool in solving the problem of representations of a given integer as a sum of squares [2]; this is the starting point for its connection to the theory of modular forms.

Later on, H. Maass considers an analogous series, motivated by his quest for the Fourier coefficients of nonanalytic modular forms. Specifically, he looks at

$$
\sum_{m=-\infty}^{\infty} \frac{1}{(\tau+m)^{\alpha}(\bar{\tau}+m)^{\beta}}
$$

where $\alpha$ and $\beta$ are complex numbers such that $\operatorname{Re}(\alpha+\beta)>1$; this requires a more complicated LSF (see [10, pp. 209-211] for a statement and proof of the formula), which was also used by Siegel [17. Both versions of Lipschitz's formula are incorporated in the following, due to John Hawkins, which first appears in [4]:

Received by the editors March 20, 2000.

2000 Mathematics Subject Classification. Primary 11F30, 11F37, 42A99.

Key words and phrases. Poisson summation formula, Lipschitz summation formula, modular forms, automorphic forms, Eisenstein series, Hecke correspondence.

(C)2001 American Mathematical Society 
Theorem 1. If $\mu \in \mathbb{R}, \operatorname{Re}(\alpha+\beta)>1$, and $\tau=x+i y \in \mathcal{H}=\{z \in \mathbb{C}: \operatorname{Im} z>0\}$, then

$$
\begin{aligned}
& \sum_{m=-\infty}^{\infty} \frac{e^{-2 \pi i \mu m}}{(\tau+m)^{\alpha}(\bar{\tau}+m)^{\beta}} \\
= & \frac{(2 \pi)^{\alpha+\beta}(-i)^{\alpha-\beta}}{\Gamma(\alpha) \Gamma(\beta)} \sum_{n=-\infty}^{\infty} a_{n+\mu}(y, \alpha, \beta) e^{2 \pi i(n+\mu) x},
\end{aligned}
$$

where $a_{n+\mu}(y, \alpha, \beta)$

$$
= \begin{cases}\Gamma(\alpha+\beta-1)(4 \pi y)^{1-\alpha-\beta} & \text { if } n+\mu=0, \\ (n+\mu)^{\alpha+\beta-1} e^{-2 \pi(n+\mu) y} \sigma(4 \pi(n+\mu) y, \alpha, \beta) & \text { if } n+\mu>0, \\ (-n-\mu)^{\alpha+\beta-1} e^{2 \pi(n+\mu) y} \sigma(-4 \pi(n+\mu) y, \beta, \alpha) & \text { if } n+\mu<0 .\end{cases}
$$

Here $\sigma(\eta, \alpha, \beta)$ denotes the special function which has the following integral representation:

$$
\int_{0}^{\infty}(u+1)^{\alpha-1} u^{\beta-1} e^{-\eta u} d u, \quad \alpha \in \mathbb{C}, \operatorname{Re} \beta>0, \operatorname{Re} \eta>0 .
$$

We remark that $\sigma(\eta, \alpha, \beta)$ is a generalization of $\Gamma(\beta)$ and that, in fact, the confluent hypergeometric function of the second kind,

$$
\Psi(\beta, \alpha+\beta ; \eta)=\frac{\sigma(\eta, \alpha, \beta)}{\Gamma(\beta)},
$$

is an entire function in $\alpha$ and $\beta$. Alternately, one can write Theorem 1 in terms of the Whittaker function instead of $\sigma(\eta, \alpha, \beta)$ (see [15]). For a simple proof of the theorem which relies on Maass's formula (i.e., on the case $\mu=0$ ) see [14, pp. 20-22]. We observe that Theorem 1 remains valid for $\mu \in \mathbb{R} \backslash \mathbb{Z}, \operatorname{Re}(\alpha+\beta)>0$. Also, note that if $\beta=0$, then the above formula does reduce to the original LSF, because $\Psi(0, \alpha ; \eta)=1$.

We have already mentioned the connection to the number-theoretic function $r_{s}(n)$, the number of ways of writing $n$ as a sum of $s$ squares. Analogously, the LSF or its generalizations may be used to find the Fourier expansion of analytic or nonanalytic Poincaré series ([8] and [4], respectively). It is therefore a crucial part of the link between entire modular forms (e.g., Eisenstein series) and certain arithmetic functions (e.g., sums of powers of divisors of an integer).

\section{NeW Summation FORMUlae}

We now present some formulae which are akin to both of those discussed above. We stress, however, that the next theorem is not a consequence of the (multivariable) Poisson summation formula.

Theorem 2 (Two-variable summation formulae). (1) If $\mu \in \mathbb{R}, \operatorname{Re}(\alpha+\beta)>1$, $\operatorname{Re} \gamma>0, \tau=x+i y \in \mathcal{H}$, and $z \in \mathcal{H}$, then

$$
\begin{aligned}
& \sum_{m=-\infty}^{\infty} \frac{e^{-2 \pi i \mu m}}{(\tau+m)^{\alpha}(\bar{\tau}+m)^{\beta}(z+m)^{\gamma}} \\
= & \frac{(2 \pi)^{\alpha+\beta+\gamma}(-i)^{\alpha-\beta+\gamma}}{\Gamma(\alpha) \Gamma(\beta) \Gamma(\gamma)} \sum_{\substack{n, \ell \in \mathbb{Z} \\
\ell+\mu>0}} \phi_{n, \ell+\mu}(\tau, z, \alpha, \beta, \gamma) e^{2 \pi i[n x+(\ell+\mu) z]},
\end{aligned}
$$


where

$$
\begin{aligned}
& \phi_{n, \ell+\mu}(\tau, z, \alpha, \beta, \gamma) \\
&=\left\{\begin{array}{rr}
e^{-2 \pi n y} \int_{0}^{b}(n+t)^{\alpha+\beta-1}(\ell+\mu-t)^{\gamma-1} e^{2 \pi i(\tau-z) t} \sigma(4 \pi(n+t) y, \alpha, \beta) d t \\
e^{2 \pi n y} \int_{0}^{b}(-n-t)^{\alpha+\beta-1}(\ell+\mu-t)^{\gamma-1} e^{2 \pi i(\bar{\tau}-z) t} \sigma(-4 \pi(n+t) y, \beta, \alpha) d t \\
\text { if } n \geq 0,
\end{array}\right. \\
& \text { if } n<0 .
\end{aligned}
$$

Here

$$
b=b(\ell+\lfloor\mu\rfloor)=\left\{\begin{array}{c}
\{\mu\} \text { if } \ell+\lfloor\mu\rfloor=0, \\
1 \quad \text { if } \ell+\lfloor\mu\rfloor \geq 1
\end{array}\right.
$$

( $\{\mu\}$ denotes the fractional part and $\lfloor\mu\rfloor$ the integer part of $\mu ; \mu=\lfloor\mu\rfloor+\{\mu\}$.

(2) If $\mu \in \mathbb{R}, \operatorname{Re}(\alpha+\beta)>1, \operatorname{Re} \gamma>0, \operatorname{Re} \delta>0$, and $\tau, z \in \mathcal{H}, y=\operatorname{Im} \tau$, then

$$
\begin{aligned}
& \sum_{m=-\infty}^{\infty} \frac{e^{-2 \pi i \mu m}}{(\tau+m)^{\alpha}(\bar{\tau}+m)^{\beta}(z+m)^{\gamma}(\bar{z}+m)^{\delta}} \\
= & \frac{(2 \pi)^{\alpha+\beta+\gamma+\delta}(-i)^{\alpha-\beta+\gamma-\delta}}{\Gamma(\alpha) \Gamma(\beta) \Gamma(\gamma) \Gamma(\delta)}\left[\sum_{n=0}^{\infty} \sum_{\ell=0}^{\infty} \sum_{p=0}^{\infty} e^{2 \pi i\left(n \tau+\left(\ell+\frac{\mu}{2}\right) z-\left(p-\frac{\mu}{2}\right) \bar{z}\right)}\right. \\
& \times \int_{\frac{\mu}{2}}^{\frac{\mu}{2}+1} \int_{\frac{\mu}{2}-1}^{\frac{\mu}{2}}(n+\{t+u\})^{\alpha+\beta-1}\left(\frac{\mu}{2}-t+\ell\right)^{\gamma-1}\left(u-\frac{\mu}{2}+p\right)^{\delta-1} \\
& +\sum_{n=1}^{\infty} \sum_{\ell=0}^{\infty} \sum_{p=0}^{\infty} e^{2 \pi i\left(-n \bar{\tau}+\left(\ell+\frac{\mu}{2}\right) z-\left(p-\frac{\mu}{2}\right) \bar{z}\right)} \\
\times & \int_{\frac{\mu}{2}}^{\frac{\mu}{2}+1} \int_{\frac{\mu}{2}-1}^{\frac{\mu}{2}}(n-\{t+u\})^{\alpha+\beta-1}\left(\frac{\mu}{2}-t+\ell\right)^{\gamma-1}\left(u-\frac{\mu}{2}+p\right)^{\delta-1} \\
& \left.\times e^{2 \pi i(\bar{\tau}\{t+u\}-t z-u \bar{z})} \sigma(4 \pi(n-\{t+u\}) y, \beta, \alpha) d t d u\right] .
\end{aligned}
$$

Proof. We present the proof of (1). First note that it suffices to consider the case where $0 \leq \mu<1$. The proof for general $\mu \in \mathbb{R}$ then follows from the simple observation that $e^{-2 \pi i \mu m}=e^{-2 \pi i\{\mu\} m}$.

Let $z=z_{1}+z_{2} i$. Since $z \in \mathcal{H}$ we have that

$$
(z+m)^{\gamma}=i^{\gamma}\left[z_{2}-\left(z_{1}+m\right) i\right]^{\gamma}
$$

where we have used the branching convention $-\pi \leq \arg (\cdot)<\pi$. Additionally, since $\operatorname{Re} \gamma>0$, we have the identity

$$
\frac{\Gamma(\gamma)}{\left[z_{2}-\left(z_{1}+m\right) i\right]^{\gamma}}=\int_{0}^{\infty} t^{\gamma-1} e^{-\left[z_{2}-\left(z_{1}+m\right) i\right] t} d t
$$


The last two statements imply that

$$
\begin{aligned}
S & =\sum_{m=-\infty}^{\infty} \frac{e^{-2 \pi i \mu m}}{(\tau+m)^{\alpha}(\bar{\tau}+m)^{\beta}(z+m)^{\gamma}} \\
& =\frac{(2 \pi)^{\gamma}(-i)^{\gamma}}{\Gamma(\gamma)} \sum_{m=-\infty}^{\infty} \frac{e^{-2 \pi i \mu m}}{(\tau+m)^{\alpha}(\bar{\tau}+m)^{\beta}} \int_{0}^{\infty} r^{\gamma-1} e^{-2 \pi\left[z_{2}-\left(z_{1}+m\right) i\right] r} d r \\
& =\frac{(2 \pi)^{\gamma}(-i)^{\gamma}}{\Gamma(\gamma)} \int_{0}^{\infty} r^{\gamma-1} e^{2 \pi i z r} \sum_{m=-\infty}^{\infty} \frac{e^{-2 \pi i \mu^{*} m}}{(\tau+m)^{\alpha}(\bar{\tau}+m)^{\beta}} d r,
\end{aligned}
$$

where $\mu^{*}=\{\mu-r\}$ is the fractional part of $\mu-r$. We emphasize that $\mu^{*}$ depends on $\mu$ and $r$. The above interchange of sum and integral is easy to justify. Since $\operatorname{Re}(\alpha+\beta)>1$ the infinite sum converges absolutely and is trivially majorized by a sum which is independent of $r$. Since $\operatorname{Re} \gamma>0$ and $z_{2}>0$ we may quote the Lebesgue dominated convergence theorem to permit the interchange.

An application of Theorem 1 tells us that the sum in the above integrand equals

$$
\begin{aligned}
& \delta\left(\mu^{*}\right) \frac{2 \pi(-i)^{\alpha-\beta} \Gamma(\alpha+\beta-1)}{\Gamma(\alpha) \Gamma(\beta)}(2 y)^{1-\alpha-\beta} \\
+ & \sum_{n+\mu^{*}>0} \frac{(2 \pi)^{\alpha+\beta}(-i)^{\alpha-\beta}\left(n+\mu^{*}\right)^{\alpha+\beta-1}}{\Gamma(\alpha) \Gamma(\beta)} \sigma\left(4 \pi\left(n+\mu^{*}\right) y, \alpha, \beta\right) e^{2 \pi i\left(n+\mu^{*}\right) \tau} \\
+ & \sum_{n+\mu^{*}<0} \frac{(2 \pi)^{\alpha+\beta}(-i)^{\alpha-\beta}\left(-n-\mu^{*}\right)^{\alpha+\beta-1}}{\Gamma(\alpha) \Gamma(\beta)} \sigma\left(-4 \pi\left(n+\mu^{*}\right) y, \beta, \alpha\right) e^{2 \pi i\left(n+\mu^{*}\right) \bar{\tau}},
\end{aligned}
$$

where

$$
\delta\left(\mu^{*}\right)= \begin{cases}0 & \text { if } 0<\mu^{*}<1 \\ 1 & \text { if } \mu^{*}=0\end{cases}
$$

But observe that

$$
\int_{0}^{\infty} r^{\gamma-1} e^{2 \pi i z r} \delta\left(\mu^{*}\right) d r=0
$$

since $\delta\left(\mu^{*}\right)=0$ almost everywhere. So we obtain

$$
\begin{aligned}
S= & \frac{(2 \pi)^{\alpha+\beta+\gamma}(-i)^{\alpha-\beta+\gamma}}{\Gamma(\alpha) \Gamma(\beta) \Gamma(\gamma)} \int_{0}^{\infty} r^{\gamma-1} e^{2 \pi i z r} \\
& \times\left[\sum_{n=0}^{\infty}\left(n+\mu^{*}\right)^{\alpha+\beta-1} \sigma\left(4 \pi\left(n+\mu^{*}\right) y, \alpha, \beta\right) e^{2 \pi i\left(n+\mu^{*}\right) \tau}\right. \\
& \left.\quad+\sum_{n=-1}^{-\infty}\left(-n-\mu^{*}\right)^{\alpha+\beta-1} \sigma\left(-4 \pi\left(n+\mu^{*}\right) y, \beta, \alpha\right) e^{2 \pi i\left(n+\mu^{*}\right) \bar{\tau}}\right] d r
\end{aligned}
$$


(N.B. although $\sigma(0, \alpha, \beta)$ is undefined, we have that $\mu^{*}=0$ only countably often, and so the nonexistence of $\sigma(0, \alpha, \beta)$ is irrelevant.) Letting

$$
A=\frac{(2 \pi)^{\alpha+\beta+\gamma}(-i)^{\alpha-\beta+\gamma}}{\Gamma(\alpha) \Gamma(\beta) \Gamma(\gamma)}
$$

and interchanging the sum and integral, we get

$$
\begin{gathered}
S=A \sum_{n=0}^{\infty} e^{2 \pi i n \tau} \int_{0}^{\infty} r^{\gamma-1} e^{2 \pi i\left(z r+\mu^{*} \tau\right)}\left(n+\mu^{*}\right)^{\alpha+\beta-1} \\
\times \sigma\left(4 \pi\left(n+\mu^{*}\right) y, \alpha, \beta\right) d r \\
+A \sum_{n=-1}^{-\infty} e^{2 \pi i n \bar{\tau}} \int_{0}^{\infty} r^{\gamma-1} e^{2 \pi i\left(z r+\mu^{*} \bar{\tau}\right)}\left(-n-\mu^{*}\right)^{\alpha+\beta-1} \\
\times \sigma\left(-4 \pi\left(n+\mu^{*}\right) y, \beta, \alpha\right) d r
\end{gathered}
$$

We substantiate the last step by noting that the integrand (prior to the interchange) is dominated by an integrable function. In fact, all but finitely many terms of the sum can be bounded independently of $r$. This follows from our assumptions and the fact that $\sigma(\eta, \operatorname{Re} \alpha, \operatorname{Re} \beta)$ is a decreasing function of $\eta$ on $(0, \infty)$.

Therefore,

$$
\begin{aligned}
S= & A \sum_{n=0}^{\infty} e^{2 \pi i n \tau} \sum_{\ell=0}^{\infty} \int_{\ell}^{\ell+1} r^{\gamma-1}\left(n+\mu^{*}\right)^{\alpha+\beta-1} \\
& \times \sigma\left(4 \pi\left(n+\mu^{*}\right) y, \alpha, \beta\right) e^{2 \pi i\left(z r+\tau \mu^{*}\right)} d r \\
& +A \sum_{n=-1}^{-\infty} e^{2 \pi i n \bar{\tau}} \sum_{\ell=0}^{\infty} \int_{\ell}^{\ell+1} r^{\gamma-1}\left(-n-\mu^{*}\right)^{\alpha+\beta-1} \\
= & A \sum_{n=0}^{\infty} \sum_{\ell=0}^{\infty} e^{2 \pi i[n \tau+(\ell+\mu) z]} \int_{\mu-1}^{\mu}(\ell+\mu-t)^{\gamma-1}(n+\{t\})^{\alpha+\beta-1} \\
& \times \sigma(4 \pi(n+\{t\}) y, \alpha, \beta) e^{2 \pi i(\tau\{t\}-z t)} d t \\
& +A \sum_{n=-1} \sum_{\ell=0}^{\infty} e^{2 \pi i[n \bar{\tau}+(\ell+\mu) z]} \int_{\mu-1}^{\mu}(\ell+\mu-t)^{\gamma-1}(-n-\{t\})^{\alpha+\beta-1} \\
& \times \sigma(-4 \pi(n+\{t\}) y, \beta, \alpha) e^{2 \pi i(\bar{\tau}\{t\}-z t)} d t,
\end{aligned}
$$

where we let $r=\ell+\mu-t$ (and so $\mu^{*}=\{\mu-r\}=\{t\}$ ). Since $0 \leq \mu<1$ (by assumption), we have that

$$
\{t\}= \begin{cases}t+1 & \text { if } \mu-1 \leq t<0 \\ t & \text { if } 0 \leq t \leq \mu\end{cases}
$$


and so we obtain

$$
\begin{gathered}
S=A \sum_{n=0}^{\infty}\left[e^{2 \pi i(n \tau+\mu z)} \int_{0}^{\mu}(\mu-t)^{\gamma-1}(n+t)^{\alpha+\beta-1}\right. \\
\times \sigma(4 \pi(n+t) y, \alpha, \beta) e^{2 \pi i(\tau-z) t} d t \\
+\sum_{\ell=1}^{\infty} e^{2 \pi i[n \tau+(\ell+\mu) z]} \int_{0}^{1}(\ell+\mu-t)^{\gamma-1}(n+t)^{\alpha+\beta-1} \\
\left.\times \sigma(4 \pi(n+t) y, \alpha, \beta) e^{2 \pi i(\tau-z) t} d t\right] \\
+A \sum_{n=-1}^{-\infty}\left[e^{2 \pi i(n \bar{\tau}+\mu z)} \int_{0}^{\mu}(\mu-t)^{\gamma-1}(-n-t)^{\alpha+\beta-1}{ }^{2 \pi(-4 \pi(n+t) y, \beta, \alpha) e^{2 \pi i(\bar{\tau}-z) t} d t}\right. \\
+\sum_{\ell=1}^{\infty} e^{2 \pi i[n \bar{\tau}+(\ell+\mu) z]} \int_{0}^{1}(\ell+\mu-t)^{\gamma-1}(-n-t)^{\alpha+\beta-1} \\
\left.\times \sigma(-4 \pi(n+t) y, \beta, \alpha) e^{2 \pi i(\bar{\tau}-z) t} d t\right] .
\end{gathered}
$$

This proves formula (1). The proof of (2) is completely analogous.

We remark that Theorem 2 could be extended to give a formula for

$$
\sum_{m=-\infty}^{\infty} e^{-2 \pi i \mu m} \prod_{j=1}^{J}\left(z_{j}+m\right)^{-\alpha_{j}}\left(\overline{z_{j}}+m\right)^{-\beta_{j}},
$$

for use with a $J$-variable Poincaré series.

\section{Applications}

3.1. Niebur modular integrals. The need for formula (1) arose naturally during the study of what the second author refers to as "nonanalytic pseudo-Poincaré series". The analysis of these functions leads to the derivation of the Fourier coefficients of Niebur modular integrals. For a detailed proof of this, see [16].

3.2. Nonanalytic automorphic forms. Let $\lambda \geq 2$ or $\lambda=2 \cos (\pi / q), q=$ $3,4,5, \ldots ; \nu_{j} \in \mathbb{Z}$ and $\gamma_{j} \in \mathbb{C}$ for $1 \leq j \leq 4$. $\mathcal{G}_{\lambda}$ will denote the Hecke group, which is generated by the translation $z \mapsto z+\lambda$ and the inversion $z \mapsto \frac{-1}{z}$. We make the usual identification of Möbius transformations with $2 \times 2$ matrices, so that these generators may be represented as

$$
S_{\lambda}=\left(\begin{array}{cc}
1 & \lambda \\
0 & 1
\end{array}\right) \text { and } T=\left(\begin{array}{cc}
0 & -1 \\
1 & 0
\end{array}\right) .
$$

Let $\alpha, \beta \in \mathbb{C}$ such that $\operatorname{Re}(\alpha+\beta)>2$ and $\alpha-\beta=\sum_{j=1}^{4} \gamma_{j} \in \mathbb{R}$. Suppose that $v_{j}: \mathcal{G}_{\lambda} \rightarrow \mathbb{C} \backslash\{0\}$ satisfies the "consistency condition":

$$
v_{j}\left(M_{3}\right)\left(c_{3} z+d_{3}\right)^{\gamma_{j}}=v_{j}\left(M_{1}\right)\left(c_{1} M_{2} z+d_{1}\right)^{\gamma_{j}} v_{j}\left(M_{2}\right)\left(c_{2} z+d_{2}\right)^{\gamma_{j}},
$$


for all $z \in \mathcal{H}$ and $M_{1}, M_{2} \in \mathcal{G}_{\lambda}$ such that $M_{1} M_{2}=M_{3}$, where $M_{k}=\left(\begin{array}{cc}* & * \\ c_{k} & d_{k}\end{array}\right)$ for $k=1,2,3$. Also put $v=\prod_{j=1}^{4} v_{j}$. Finally, write $v_{j}\left(S_{\lambda}\right)=e^{2 \pi i \kappa_{j}}, 0 \leq \operatorname{Re} \kappa_{j}<1$. Define

$$
f(z)=\sum_{M_{c, d}} \frac{e^{\frac{2 \pi i}{\lambda}\left[\left(\nu_{1}+\kappa_{1}\right) M z+\left(\nu_{2}+\kappa_{2}\right) M \bar{z}+\left(\nu_{3}+\kappa_{3}\right) M(-z)+\left(\nu_{4}+\kappa_{4}\right) M(-\bar{z})\right]}}{v(M)(c z+d)^{\alpha}(c \bar{z}+d)^{\beta}},
$$

$z \in \mathcal{H}$, where the sum is over $M=M_{c, d} \in\left\langle S_{\lambda}\right\rangle \backslash \mathcal{G}_{\lambda}$, that is, over all matrices in $\mathcal{G}_{\lambda}$ with distinct lower rows.

In [12], the first author showed the following:

Theorem 3. (i) $f(z+\lambda)=v\left(S_{\lambda}\right) f(z)$;

(ii) $f$ is absolutely uniformly convergent on

$$
\mathcal{H}_{\varepsilon}=\left\{z \in \mathbb{C}: \operatorname{Im} z>\varepsilon>0,|\operatorname{Re} z|<\frac{1}{\varepsilon}\right\},
$$

(i.e., the absolute series is uniformly convergent on $\mathcal{H}_{\varepsilon}$ ) for $\varepsilon>0$;

(iii) $f$ is a real analytic function in $z$ and $\bar{z}$ for $\operatorname{Im} z>\varepsilon$;

(iv) $f$ satisfies the transformation law

$$
z^{-\alpha} \bar{z}^{-\beta} f(-1 / z)=v(T) f(z),
$$

for $\operatorname{Im} z>0$.

However, $f$ is not an automorphic form, no matter which of the many competing definitions of "form" we abide by, for it lacks the proper expansion. This is easy to see by summation formula (2), following the usual type of calculation; for general $v_{j}, \nu_{j}$, the expansion of $f(z)$ involves terms whose coefficients are sums of definite double integrals of confluent hypergeometric functions in $\operatorname{Im} z$. This is a far cry from the functions usually considered to be nonanalytic forms, for example in [11.

An important special case of $f$, which appears in [10, is the Maass nonanalytic Eisenstein series (this is the case $\lambda=1, \alpha-\beta \in 2 \mathbb{Z}, v_{j} \equiv 1$ and $\nu_{j}=0 \forall j$ ); it suggests a possible generalization of the so-called Riemann-Hecke-Bochner correspondence for nonanalytic automorphic integrals (see [13]). Any space which contains the Maass series and is closed under certain commonly used weight-changing operators (see [12]) should allow Fourier coefficients so that a typical term has the form

$$
y^{w} \sigma(4 \pi n y, \alpha, \beta) e^{-2 \pi n y},
$$

where $n$ is a positive integer and $w$ is complex. But straightforward calculation using identities in $[3$ shows that the Mellin transform of this last expression is

$$
(2 \pi n)^{-s-w} \Gamma(s+w) B(\beta ; 1-\alpha-\beta+s+w){ }_{2} F_{1}(s+w, \beta ; 1-\alpha+s+w ;-1) .
$$

( $B$ and ${ }_{2} F_{1}$ denote the beta and hypergeometric functions, respectively, using the standard definitions in [7]). A more precise formulation of the Hecke correspondence for such functions has, so far, proved elusive.

\section{REFERENCES}

[1] Benedetto, J.J. and Zimmerman, G. (1997). Sampling Multipliers and the Poisson Summation Formula. J. of Fourier Analysis and Appl. 3, 505-524. MR 99b:42011

[2] Dickson, L.E. (1930). Studies in the Theory of Numbers. Univ. of Chicago Press, Chicago. 
[3] Erdelyi, A. (1981). Higher Transcendental Functions, Vol. I. Krieger Publishing Company, Melbourne, Florida. MR 84h:33001a

[4] Knopp, M.I. (1989). On the Fourier Coefficients of Cusp Forms Having Small Positive Weight. Proc. of Symposia in Pure Math. 49 (2), 111-127. MR 90j:11035

[5] Knopp, M.I. (1993). Modular Functions in Analytic Number Theory, 2nd edition. Chelsea, New York.

[6] Knopp, M.I. and Robins, S., Easy Proofs of Riemann's Functional Equation for $\zeta(s)$ and of Lipschitz Summation. Proc. Amer Math. Soc., posted on February 2, 2001, PII S 00029939(01)06033-6 (to appear in print).

[7] Lebedev, N.N. (1972). Special Functions and Their Applications. Dover, New York. MR 50:2568

[8] Lehner, J. (1964). Discontinuous Groups and Automorphic Functions. Amer. Math. Soc., Providence, RI. MR 29:1332

[9] Lipschitz, R. (1889). Untersuchung der Eigenschaften einer Gattung von unendlichen Reihen. J. Reine und Angew. Math. CV, 127-156.

[10] Maass, H. (1964). Modular Functions of One Complex Variable, Tata Institute, Bombay. MR 36:1392

[11] Pasles, P.C. (1999). Nonanalytic automorphic integrals on the Hecke groups. Acta Arith. 90 (2), 155-171. MR 2000i:11074.

[12] Pasles, P.C. (2000). Convergence of Poincaré series with two complex coweights. Contemp. Math., 251, AMS, Providence, 453-461.

[13] Pasles, P.C. (2000). A Hecke correspondence theorem for nonanalytic automorphic integrals. J. Number Theory 83 (2), 256-281. CMP 2000:16

[14] Pribitkin, W. (1995). The Fourier coefficients of modular forms and modular integrals having small positive weight. Doctoral Dissertation, Temple University, Philadelphia.

[15] Pribitkin, W. (1999). The Fourier coefficients of modular forms and Niebur modular integrals having small positive weight, I. Acta Arith. 91 (4), 291-309. CMP 2000:07

[16] Pribitkin, W. (2000). The Fourier coefficients of modular forms and Niebur modular integrals having small positive weight, II. Acta Arith. 93 (4), 343-358. CMP 2000:13

[17] Siegel, C.L. (1956). Die Funktionalgleichungen einiger Dirichletscher Reihen. Math. Z. 63, 363-373. MR 17:602c

Department of Mathematical Sciences, Villanova University, Villanova, PennsylvaNIA 19085

E-mail address: pasles@member.ams.org

Department of Mathematics, Princeton University, 607 Fine Hall, Princeton, New JERSEY 08544

E-mail address: w_pribitkin@msn.com

E-mail address: wladimir@princeton.edu 